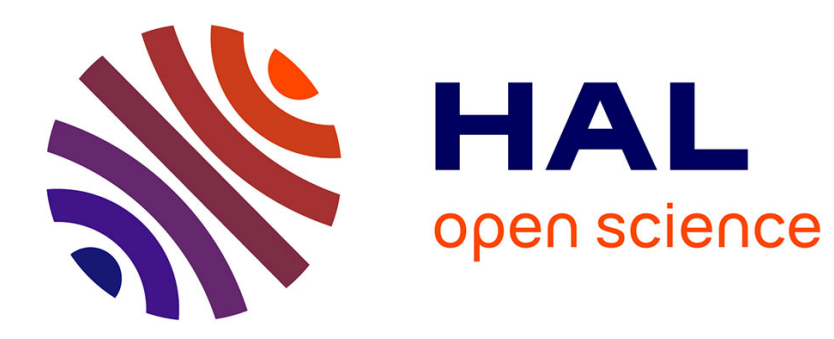

\title{
MÉTHODES TEMPS-FRÉQUENCE EN ACOUSTIQUE
}

\author{
P. Flandrin, J. Sessarego
}

\section{To cite this version:}

P. Flandrin, J. Sessarego. MÉTHODES TEMPS-FRÉQUENCE EN ACOUSTIQUE. Journal de Physique Colloques, 1990, 51 (C2), pp.C2-707-C2-716. 10.1051/jphyscol:19902165 . jpa-00230468

\section{HAL Id: jpa-00230468 https://hal.science/jpa-00230468}

Submitted on 1 Jan 1990

HAL is a multi-disciplinary open access archive for the deposit and dissemination of scientific research documents, whether they are published or not. The documents may come from teaching and research institutions in France or abroad, or from public or private research centers.
L'archive ouverte pluridisciplinaire HAL, est destinée au dépôt et à la diffusion de documents scientifiques de niveau recherche, publiés ou non, émanant des établissements d'enseignement et de recherche français ou étrangers, des laboratoires publics ou privés. 
COLLOQUE DE PHYSIQUE

Colloque C2, supplément au $n^{\circ} 2$, Tome 51, Février 1990

ler Congrès Françats d'Acoustique 1990

\author{
MÉTHODES TEMPS-FREQUENCE EN ACOUSTIQUE
}

\author{
P. FLANDRIN Ot J.P. SESSAREGo* \\ Laboratoire de Traitement du Signal (URA 346 CNRS), ICPI, 25 Rue du \\ Plat, F-69288 Lyon Cedex 02, France \\ * CNRS, Laboratoire de Mécanique et d'Acoustique, 31 Chemin J. Aiguier, \\ F-13402 Marseille Cedex 9. France
}

Résumé - Le développement actuel des méthodes temps-fréquence en fait un outil de choix pour l'analyse et le traitement des signaux acoustiques. Après une présentation générale des distributions (énergétiques et non-paramétriques) envisageables, on souligne l'intérêt de la distribution de WignerVille et on en discute les propriétés. L'utilité des méthodes temps-fréquence est illustrée sur quelques exemples et par une revue de la littérature concernant leurs applications dans des domaines variés de l'acoustique (acoustique physique, propagation, parole, bioacoustique, électroacoustique, ...).

\begin{abstract}
Owing to their recent development, time-frequency methods are currently gaining an increasing interest for the analysis and processing of acoustic signals. After a general presentation of possible (energetic and non-parametric) approaches, emphasis is put on the Wigner-Ville distribution and its most useful properties. Usefulness of time-frequency methods is illustrated on some examples and supported by a review of the literature concerning their effective applications to various fields of acoustics (physical acoustics, propagation, speech, bioacoustics, electroacoustics, ...).
\end{abstract}

\title{
INTRODUCTION
}

Depuis maintenant une dizaine d'années, un grand nombre de travaux ont été consacrés au développement de nouvelles méthodes d'analyse temps-fréquence et à leurs applications. Parmi celles-ci figurent en bonne place des problèmes liés à des domaines très variés de l'acoustique: ceci est évidemment dû à la grande richesse des signaux rencontrés en acoustique et à leurs fortes non-stationnarités. D'une manière très générale, on peut dire que l'introduction de méthodes temps-fréquence répond au souci d'étendre les outils spectraux classiques (dont on sait qu'ils sont pertinents dans des cas stationnaires) à des situations évolutives, i.e. de réconcilier fréquence et non-stationnarité. Du paradoxe que 1) l'essentiel des méthodes classiques sont destinées aux cas stationnaires et que 2) l'essentiel des signaux «réels» sont non-stationnaires (l'information la plus grande se concentrant d'ailleurs sur les non-stationnarités), naît le besoin de descriptions mixtes susceptibles de représenter conjointement un signal, et dans sa décomposition spectrale, et dans sa chronologie.

Des approches systématiques pour construire des représentations temps-fréquence ont maintenant succédé aux outils empiriques ou ad hoc développés historiquement au fil des besoins d'applications spécifiques. Ne pouvant prétendre ici à l'exhaustivité, nous présentons en $\S$ I une démarche rationnelle pour justifier du choix d'une représentation temps-fréquence dans un contexte déterministe, énergétique et non-paramétrique, renvoyant à [T12] pour une présentation d'autres facettes du problème (aléatoire et non-paramétrique dans [T9]; aléatoire et paramétrique dans [T10]). On trouvera ensuite en $\S$ II quelques exemples d'illustration ainsi qu'un panorama assez large d'applications effectives de méthodes temps-fréquence dans différents domaines de l'acoustique.

\section{I - METHODES TEMPS-FREQUENCE}

\section{A. Représentations énergétiques non-paramétriques}

Une fois reconnue l'importance d'une représentation temps-fréquence, se pose le problème du choix de cette dernière. Ce problème se pose car, contrairement au cas d'une description fréquentielle indépendante du temps pour lequel la transformation de Fourier est l'unique solution, il apparaît que, dans le cas où l'on recherche une description fréquentielle dépendante du temps, une multiplicité de solutions est offerte, chacune avec des avantages et des inconvénients propres [T3, T6, T12]. Une restriction naturelle, sur laquelle l'accent sera mis dans cette présentation, est de s'intéresser à des distributions énergétiques et non-paramétriques de façon à pouvoir aborder l'analyse de signaux pour lesquels on ne dispose pas nécessairement de modèles $a$ priori.

1. Spectrogramme et sonagramme. Avant d'aborder le problème dans sa généralité, on peut envisager une solution intuitive qui parte, en l'adaptant à une situation dépendante du temps, de l'analyse classique indépendante du temps: la transformation de Fourier. L'idée est alors de considérer une situation non-stationnaire comme la concaténation de situations quasi-stationnaires pour lesquels l'analyse de Fourier est pertinente. Ainsi, au lieu d'associer à un signal temporel $x(t)$ sa seule densité d'énergie fréquentielle: 


$$
\Gamma_{x}(v)=\left|\int_{-\infty}^{+\infty} x(t) \mathrm{e}^{-\mathrm{i} 2 \pi v t} d t\right|^{2},
$$

quantité indépendante du temps, on peut imaginer de construire une succession de telles grandeurs locales obtenues en observant le signal total à travers une fenêtre (a court-terme) glissante $h(t)$ :

$$
S_{x}(t, v)=\left|\int_{-\infty}^{+\infty} x(u) h^{*}(u-t) \mathrm{e}^{-\mathrm{i} 2 \pi v u} d u\right|^{2} .
$$

On obtient bien ainsi une description fréquentielle dépendante du temps s'apparentant à une analyse spectrale évolutive. Néanmoins, une telle représentation (appelée spectrogramme ou sonagramme [T1, T3, T11]) possède des limitations dues essentiellement à l'arbitraire de la fenêtre d'analyse $h(t)$. En effet, pour obtenir une bonne résolution temporelle, il est nécessaire de travailler avec une fenêtre temporelle courte, ce qui malheureusement conduit à une résolution fréquentielle pauvre, du fait de l'opération de transformation de Fourier. Réciproquement, une bonne résolution fréquentielle nécessite une fenêtre d'analyse longue, ce qui, en retour, correspond à une mauvaise résolution temporelle. Ainsi, un spectrogramme est par nature confronté à un compromis entre ses comportements temporel et fréquentiel, ce qui nécessite de chercher d'autres solutions, moins intuitives peut-être, mais plus performantes.

2. Classe générale. Si l'on pose le problème d'associer à un signal temporel une représentation bilinéaire, de façon à distribuer son énergie dans le plan temps-fréquence, on peut montrer [T6] que la seule contrainte d'invariance par translations (i.e. que la distribution obtenue «suive» les translations en temps et/ou en fréquence du signal analysé) est suffisante pour réduire la classe des solutions admissibles à la seule classe, dite de Cohen, dont l'expression s'écrit [T3]:

$$
\begin{gathered}
C_{x}(t, v, I)=\int_{-\infty}^{+\infty} \int_{-\infty}^{+\infty} W_{x}(u, n) \Pi(u-t, n-v) d u d n \\
W_{x}(t, v)=\int_{-\infty}^{+\infty} x\left(t+\frac{\tau}{2}\right) x^{*}\left(t-\frac{\tau}{2}\right) \mathrm{e}^{-\mathrm{i} 2 \pi v \tau} d \tau
\end{gathered}
$$

est la distribution de Wigner-Ville [T2, T5] et où $\Pi$ est une fonction temps-fréquence arbitraire.

Le fait remarquable est que la formulation (3) permet, par un choix judicieux de la fonction arbitraire, de retrouver comme cas particuliers la quasi-totalité des distributions proposées par différents auteurs depuis une quarantaine d'années [T2, T3, T6]. De plus, on dispose ainsi d'une caractérisation permettant de sélectionner des représentations admissibles relativement à des contraintes imposées a priori. La conclusion de nombreuses études [T6], menées depuis maintenant une dizaine d'années, est que la représentation satisfaisant au plus grand nombre de contraintes envisageables en Traitement du Signal est la distribution de Wigner-Ville (4). Nous allons maintenant en détailler les caractéristiques les plus intéressantes, en vue d'applications en acoustique.

3. Distribution de Wigner-Ville. Sans entrer dans trop de détails, on peut dresser une liste de propriétés de la distribution de Wigner-Ville (DWV), qui se déduisent de sa définition et montrent son intérêt (se reporter, e.g., à $[\mathrm{T} 5, \mathrm{~T} 6])$.

1) La DWV est un «déploiement» temporel de la densité spectrale d'énergie puisque:

$$
\int_{-\infty}^{+\infty} W_{x}(t, v) d t=\Gamma_{x}(v)
$$

Ceci permet de préserver l'information globale liée à un spectre (significatif par exemple de résonances) tout en lui associant une chronologie.

2) De même, la DWV peut être considérée, à tout instant, comme une décomposition spectrale de la puissance instantanée puisque:

$$
\int_{-\infty}^{+\infty} W_{x}(t, v) d v=|x(t)|^{2}
$$

3) En conséquence des points 1) et 2), la DWV est bien une distribution de l'énergie du signal dans le plan temps-fréquence. 
4) Outre des informations de module (comme celles des eqs.(5) et (6)), des informations de phase sont aussi présentes dans la DWV. En particulier, le retard de groupe s'obtient naturellement comme lieu des centres de gravité de la DWV dans le plan temps-fréquence:

$$
\left[\int_{-\infty}^{+\infty} t W_{x}(t, v) d t\right] /\left[\int_{-\infty}^{+\infty} W_{x}(t, v) d t\right]=-\frac{1}{2 \pi} \frac{d}{d t} \arg (X(v)\} .
$$

Cette propriété est particulièrement importante pour l'utilisation d'une représentation temps-fréquence comme outil d'estimation de la dispersion de vitesse d'un milieu de propagation ( $c f$. \& II-A.1 et 2). On peut, de manière similaire, accéder de façon exacte à la loi de fréquence instantanée d'un signal modulé.

5) La DWV conserve les supports temporel et fréquentiel d'un signal et permet donc une localisation précise à la fois en temps et en fréquence. C'est là encore une propriété fondamentale pour pouvoir séparer commodément différents événements dans le plan temps-fréquence.

6) La DWV préserve la structure convolutive d'entrée-sortie des filtres linéaires, tout en y adjoignant une dépendance fréquentielle explicite :

$$
y(t)=\int_{-\infty}^{+\infty} x(u) h(t-u) d u \Rightarrow W_{y}(t, v)=\int_{-\infty}^{+\infty} W_{x}(u, v) W_{h}(t-u, v) d u .
$$

7) La DWV est reliée à la fonction d'ambiguitté $A_{x}(n, \tau)$ [T3], qui est une mesure de corrélation tempsfréquence, par une relation de transformation de Fourier bidimensionnelle:

$$
A_{x}(n, \tau)=\int_{-\infty}^{+\infty} x\left(u+\frac{\tau}{2}\right) x^{*}\left(u-\frac{\tau}{2}\right) \mathrm{e}^{\mathrm{i} 2 \pi n u} d u=\int_{-\infty}^{+\infty} \int_{-\infty}^{+\infty} W_{x}(t, v) \mathrm{e}^{\mathrm{i} 2 \pi(n t+\tau v)} d t d v
$$

Ceci permet d'envisager les problèmes classiques faisant intervenir la fonction d'ambiguïté (en particulier détection-estimation optimales, filtrage adapté et synthèse de signaux) d'une façon duale en travaillant directement dans le plan temps-fréquence avec la DWV [T8].

L'ensemble de ces propriétés fait de la DWV un outil privilégié pour l'analyse des signaux évolutifs. Son utilisation pratique doit néanmoins incorporer quelques modifications apportées à la définition (4) de façon à réduire l'effet de deux propriétés indésirables de la DWV: l'existence de valeurs négatives locales et d'interférences entre composantes.

4. Interprétation et modifications. En conséquence de sa structure bilinéaire, la DWV crée naturellement des termes d'interférences entre les différentes composantes d'un signal. Ces termes, qui sont par certains côtés utiles puisqu'ils portent une information sur les relations existant entre les différentes composantes, sont en général perturbateurs vis-à-vis de la lisibilité d'un diagramme temps-fréquence. On peut en effet montrer qu'ils sont caractérisés par une structure oscillatoire, à l'inverse des termes «signal» au comportement plus adouci [T6]. Cette différence de comportement suggère une modification naturelle à la définitión (4) pour diminuer l'influence des termes d'interférence: il suffit de lisser la DWV dans le plan temps-fréquence, c'est-à-dire d'utiliser une représentation du type (3) dans laquelle la fonction arbitraire est du type filtrage passe-bas.

Si l'on examine simultanément les eqs.(2) et (3), on peut montrer qu'en fait le spectrogramme lui-même est un cas particulier de la classe de Cohen obtenu en choisissant comme fonction arbitraire la DWV de la fenêtre d'analyse :

$$
S_{x}(t, v)=C_{x}\left(t, v, W_{h}\right)=\int_{-\infty}^{+\infty} \int_{-\infty}^{+\infty} W_{x}(u, n) W_{h}(u-t, n-v) d u d n
$$

Les limitations évoquées au § I-A.1 s'interprètent maintenant en observant que la fonction utilisée pour.le lissage est elle-même une DWV, et est donc de supports temporel et fréquentiel identiques à ceux de la fenêtre d'analyse $h(t)$. Une amélioration naturelle est d'utiliser les deux degrés de liberté offerts par les deux dimensions du plan temps-fréquence en choisissant comme fonction de lissage une fonction séparable en ses deux variables. Ceci conduit à la définition d'une distribution pseudo-Wigner-Ville lissée (DPWVL) [T5, T6]:

$\Pi_{\mathrm{S}}(t, v)=g(t) H(v) \Rightarrow C_{x}\left(t, v, \Pi_{\mathrm{S}}\right)=\int_{-\infty}^{+\infty} \int_{-\infty}^{+\infty} W_{x}(u, n) \mathrm{g}(u-t) H(n-v) d u d n$ 


$$
=\int_{-\infty}^{+\infty} h(\tau)\left[\int_{-\infty}^{+\infty} g(u-t) x\left(u+\frac{\tau}{2}\right) x^{*}\left(u+\frac{\tau}{2}\right) d u\right] \mathrm{e}^{-\mathrm{i} 2 \pi v \tau} d \tau
$$

Il importe de noter que, dans une telle représentation, la fenêtre d'analyse $h(t)$ ne contrôle plus que la résolution fréquentielle, la résolution temporelle étant quant à elle contrôlée par la fenêtre de lissage $g(t)$. Ces deux grandeurs peuvent donc être réglées de manière indépendante, ce qui permet de dépasser le compromis inhérent au spectrogramme. D'une manière plus précise, le compromis auquel le spectrogramme est confronté se situe entre résolution temporelle et résolution fréquentielle alors que celui de la DPWVL se situe entre la finesse de description des composantes individuelles et limportance des structures interférentielles, i.e. entre résolution conjointe et lisibilité.

\section{B. Autres approches}

1. Transformée en ondelettes. L'approche suivie précédemment, et qui a permis de mettre en avant la DWV, est énergétique et non-paramétrique, ce qui signifie qu'elle réalise une distribution de l'énergie du signal dans le plan temps-fréquence, sans modèle de structure a priori. D'autres approches sont cependant envisageables, qui sont destinées à décomposer le signal lui-même sur un ensemble de «briques de base» aussi localisées que possible dans le plan temps-fréquence. Si toutes les briques de la décomposition se déduisent d'une brique unique par translations temporelles et fréquentielles, on a affaire à une décomposition de type Gabor-Helström s'apparentant à une transformation de Fourier à court-terme et en présentant l'essentiel des limitations ( $c f$. $\$$ I-A.1 et I-A.4). Si le pavage du plan est maintenant assuré par des translations et changements d'échelle (dilatation ou compression) de la brique de base le long de l'axe du temps, on obtient une transformée en ondelettes [T4]. Si cette approche présente un certain intérêt dans le cas d'applications spécifiques bien adaptées à la structure d'échelle de la transformée ( $c f$. $\S \mathrm{II})$, il faut néanmoins noter que d'un point de vue énergétique (i.e. de localisation dans le plan), une relation de lissage analogue à (10) existe pour le module carré de la transformée en ondelettes (appelé scalogramme) [T7], le lissage étant fonctionnellement dépendant de la bande de fréquence analysée. Les limitations de résolution conjointe mentionnées pour le spectrogramme s'appliquent donc, mutatis mutandis, au scalogramme.

2. Méthodes paramétriques. La décomposition d'un signal en composantes élémentaires peut être vue comme une modélisation avec peu d'a priori, ce qui conduit à des descriptions par l'intermédiaire d'un grand nombre de paramètres. Cependant, si l'on possède une connaissance a priori sur la structure possible des signaux à analyser, il est clair qu'une représentation paramétrique construite sur un modèle s'en déduisant sera parcimonieuse et pertinente. On trouvera dans [T10] une présentation générale de la construction de représentations tempsfréquence paramétriques dans un contexte non-stationnaire. De manière assez analogue au cas non-paramétrique, on peut envisager deux types d'approches. La première (approche adaptative) est liée à une approximation quasistationnaire et revient, e.g., à remplacer (2) par un spectre ARMA stationnaire local. La deuxième (approche évolutive) ne fait pas d'hypothèse de quasi-stationnarité et incorpore les non-stationnarités dans le modèle (e.g. ARMA à coefficients dépendant du temps).

\section{II - DOMAINES D'APPLICATION}

\section{A. Acoustique physique et propagation}

1. Ondes dispersives, sismique et vibrations. Une situation simple pouvant motiver l'utilisation d'une analyse temps-fréquence est celle de la propagation d'ondes dispersives. En effet, si une impulsion est émise dans un milieu pour lequel les différentes fréquences ne se propagent pas à la même vitesse, un diagramme tempsfréquence fournit directement, par la localisation de son énergie, l'information relative à la loi de dispersion. De plus, d'après (7), une estimation exacte de cette quantité est possible si l'on utilise pour l'analyse une DWV. C'est ce qui est illustré en Fig. 1 dans le cas d'ondes de plaques [O8].

Un domaine pour lequel se posent des problèmes d'estimation de dispersion de vitesse et d'atténuation d'ondes élastiques est celui de la sismique et de l'exploration géophysique. On trouvera dans [O1] une présentation de l'intérêt de la DWV dans un tel contexte, et dans [O2] une extension (de type interspectral) de cette méthode destinée à comparer directement dans le plan temps-fréquence un signal vibrosismique émis à ses multiples réflexions sur les différentes couches du sous-sol. Par ailleurs, deux approches très intéressantes au problème de la séparation d'ondes sismiques se recouvrant simultanément en temps et en fréquence sont proposées dans [O3]: la première repose sur l'utilisation de la DWV comme outil d'analyse suivi de re-synthèse partielle et itération après élimination du résidu; la deuxième fait usage d'une reconnaissance de structures tempsfréquence connues (a priori ou par apprentissage) par calcul d'une DWV croisée. D'un point de vue historique, il 
est intéressant de noter que ce sont également des motivations de géophysique interne et de sismique qui sont à lorigine de la transformation en ondelettes [O7].

Un domaine connexe est celui des vibrations pour lequel on peut citer des travaux relatifs à l'utilisation de la DWV pour des problèmes comme la détection de défauts de fonctionnement de moteurs [O4], la caractérisation fine de signaux transitoires associés à des chocs de piston [O5] ou encore l'aide à la modélisation de signaux de bruit neutronique [06].

2. Diffusion acoustique. La caractérisation de cibles géométriques de forme simple à partir d'échos en rétrodiffusion (réponses impulsionnelles acoustiques) est un autre exemple pour lequel une approche tempsfréquence se révèle intéressante [D1-D3]. En effet, ce problème nécessite d'utiliser des techniques adaptées aux différentes structures du signal porteuses des informations recherchées, structures qui sont essentiellement de trois types :

1) temporelles: écho spéculaire, arrivées successives d'ondes circonférentielles ou d'ondes de surface;

2) fréquentielles : résonances acoustiques liées à des interférences d'ondes de surface;

3) conjointes : dispersion de vitesse des différentes ondes, se manifestant par des temps de parcours différents suivant la fréquence.

Pour le point 1), on peut imaginer de travailler directement sur le signal temporel en sortie de capteur. La difficulté est qu'alors il est difficile, voire impossible, de séparer des composantes de contenus fréquentiels différents si elles se recouvrent, même partiellement, en temps. Réciproquement, pour le point 2), une analyse fréquence par fréquence est envisageable, mais elle présente linconvénient de supprimer toute notion de chronologie. Dans ces deux cas, et a fortiori pour le point 3), une méthode d'analyse temps-fréquence permet d'accéder de façon simultanée aux différentes grandeurs mentionnées, qui sont également nécessaires. Un exemple d'application de trois méthodes différentes (spectrogramme, DPWVL et scalogramme) à l'analyse d'un écho (en rétrodiffusion) de coque sphérique mince est présenté en Fig. 2. Toutes trois font apparaître, outre l'écho spéculaire (caractérisé par une faible durée et une bande large), deux réseaux d'ondes superposés, dont les vitesses moyennes et les dispersions sont différentes. On distingue en outre un motif basse-fréquence associé à un mode de coque. La meilleure localisation simultanée (en temps et en fréquence) de ces différentes composantes est assurée par l'approche basée sur la DWV, mais au prix de l'existence de structures interférentielles. A l'inverse, l'utilisation d'un spectrogramme ou d'un scalogramme réduit les interférences entre composantes, mais au prix d'une moindre résolution sur chacune d'elles. Cet exemple souligne en fait quelques unes des spécificités des différentes analyses et l'intérêt que peut présenter leur utilisation complémentaire.

3. Milieux réverbérants. Deux outils classiques pour l'étude des milieux réverbérants (en particulier en acoustique sous-marine) sont la fonction d'ambiguïté et la fonction de diffusion [R1]. On peut en fait montrer que des descriptions équivalentes sont possibles dans le plan temps-fréquence à l'aide de la DWV [T8] ou éventuellement du spectrogramme [T1]. Une approche par DWV est suivie dans [R5] pour l'étude de la réverbération associée à la rétrodiffusion d'un milieu en mouvement.

Un autre domaine dans lequel interviennent des problèmes de réverbération est celui de l'acoustique des salles. On trouvera dans [R2] une utilisation de la fonction d'ambiguïté dans ce contexte inhabituel et, dans [R4], une utilisation de la DWV. Dans cette dernière étude, consacrée à l'évaluation de haut-parleurs dans un environnement naturel, l'originalité consiste à mesurer une fonction de transfert locale (spatialement) en luttant contre les trajets multiples par un moyennage de DWV associées à des positions de microphones voisines. Pour ce problème de séparation de chemins multiples, une autre solution possible serait d'utiliser la méthode dite TDS (Time Delay Spectrometry), qui fait usage d'un signal modulé linéairement en fréquence: celle-ci se révèle en fait être équivalente au calcul d'un spectrogramme. On trouvera dans [R3] une discussion des liens existant entre TDS, spectrogramme et DWV.

\section{B. Communication}

1. Parole. Par sa richesse en non-stationnarités, le signal de parole semble a priori un bon candidat pour l'utilisation de méthodes temps-fréquence. D'une certaine façon, c'est lui qui se trouve à la source des méthodes standard couramment utilisées, comme le spectrogramme et le sonagramme. Depuis les premiers travaux [T11, P9], de nombreuses caractérisations de signaux de parole ont reposé sur ces outils qui restent encore d'un très grand intérêt et pour lesquels une expertise humaine peut aller jusqu'à la reconnaissance [P16]. Parmi les extensions et améliorations apportées à la définition de base, on peut noter l'approche développée dans [P5], qui utilise la phase de la transformation de Fourier à court-terme et représente le signal de parole dans un plan tempsfréquences instantanées: il en résulte une meilleure localisation des formants et une plus grande continuité dans leur représentation. Une extension différente du sonagramme classique est celle proposée dans [P10] où il est fait usage de lissages locaux d'orientation quelconques dans le plan, et pouvant s'adapter, e.g., au suivi d'évolutions formantiques. Une interprétation de cette approche repose en fait sur la généralisation de (10) par utilisation de noyaux de lissage directionnels sur la DWV.

Une des premières utilisations explicites de la DWV pour l'analyse de la parole [P3] a consisté en une application directe de la définition, ce qui a conduit à des images difficilement interprétables du fait de la grande richesse des signaux analysés et des interférences multiples entre les composantes les constituant. Des 
améliorations ont alors été apportées à cette approche dans deux directions complémentaires: la première a été de reconnaître que la DWV présentait un intérêt essentiel pour lanalyse des structures fines du signal de parole; la deuxième a été de systématiser l'usage de lissages souples de la DWV, en particulier à l'aide de fonctions séparables. On trouvera dans [P6] et [P12-P13] une approche au problème de la reconnaissance de consonnes et de l'extraction d'invariants, dans [P14-P15] une mise en évidence simultanée de la structure temporelle (pitch) et frequentielle (formants) d'un segment de voyelle ainsi que de la signature de plosives et de fricatives, dans [P11] une méthode de détermination de l'instant de fermeture glottale et dans [P2] une bonne reconnaissance monosyllabique. Ces différentes études font toutes usage de versions lissées (de façon séparable) de la DWV. On trouvera par ailleurs dans [P4] une comparaison entre plusieurs méthodes temps-fréquence (Wigner-Ville, Rihaczek et Page) dans quelques cas simples. A titre d'illustration, la Fig. 3 présente d'une part une analyse à basse résolution conjointe (spectrogramme) d'un segment de parole et, d'autre part, une analyse à haute résolution conjointe (DPWVL) d'un détail dè ce même segment.

$\mathrm{Du}$ fait de la structure à surtension constante observée (en première approximation) dans le système auditif, les méthodes temps-échelle sont $a$ priori attrayantes pour l'analyse de la parole. Des premières tentatives d'utilisation de la transformation en ondelettes ont été faites, par exemple pour le problème de la modification de la durée sans changement fréquentiel ou de la transposition sans modification de durée [T4]. On trouvera par ailleurs dans [P1] et [P7] des méthodes d'inspiration proche.

L'accent ayant été mis, dans cette présentation des méthodes temps-fréquence, sur les approches nonparamétriques, nous ne ferons que mentionner les travaux relatifs aux modèles évolutifs ou adaptatifs en renvoyant à [T10] pour plus de détails. La possible adéquation des modèles utilisés à la structure des signaux de parole conduit alors évidemment à des descriptions parcimonieuses et pertinentes, ce qui en fait des outils de choix pour de la reconnaissance ou de la synthèse. L'emploi de modèles évolutifs permet en particulier de traiter globalement des séquences complexes, sans nécessairement travailler à l'échelle du phonème.

2. Acoustique musicale. Ainsi que beaucoup d'auteurs l'ont remarqué, la notation musicale est un précurseur des représentations temps-fréquence. En fait, compte tenu de la répartition effective des hauteurs sur une portée, c'est plutôt de représentation temps-échelle qu'il faudrait parler. Ceci explique que plusieurs travaux ont porté sur l'emploi de telles méthodes, qu'il s'agisse de transformées en ondelettes (non-orthogonales [M4-M5] ou orthogonales [M2]) ou de méthodes apparentées [M6]. Certains auteurs ont cependant mis en cuvre des outils temps-fréquence proprement dit, comme la DWV pour la caractérisation de transitoires d'attaques dans la flûte [M1], ou une variante de l'analyse de Fourier à court-terme (appelée Analyse Spectrale Différentielle [M3]) pour l'étude de sons multiphoniques.

3. Bioacoustique. Une première utilisation de méthodes temps-fréquence en bioacoustique est qualitative et, d'une certaine façon, semblable à celle utilisée en parole après l'invention du sonagramme: il s'agit essentiellement de caractériser, par une structure temps-fréquence, les signaux de communication émis par divers animaux (oiseaux, singes, ...) [B8]. A quelques exceptions près (comme la caractérisation de vocalisations de grenouilles par utilisation de la distribution de Rihaczek [B6] ou la détection acoustique d'aérophagies surcritiques par transformation en ondelettes [B7]), la plupart des auteurs ne font usage que de spectrogrammes ou de sonagrammes.

L'utilisation de représentations plus fines se rencontre par contre dans l'étude des émissions sonar des chauves-souris et de certains mammiferes marins [B10]. On trouvera dans [B4, B9] des exemples d'utilisation de la DWV et dans [B5] l'intérêt de modèles évolutifs dans un tel contexte. Il se trouve en fait que, dans le cas de la chauve-souris, les méthodes temps-fréquence peuvent alors être l'objet d'une deuxième utilisation: fournir un modèle explicatif de traitement des échos reçus, qui soit à la fois en accord avec les expériences de laboratoire [B11] et avec les contraintes neurophysiologiques [B 10]. Ceci fournit un exemple de base biologique à des réalisations de traitements optimaux par corrélations de structures temps-fréquence, dont l'importance théorique est discutée dans $[\mathrm{T} 1, \mathrm{~T} 8]$.

\section{C.Divers}

1. Electroacoustique et instrumentation. Une des premières applications effectives de la DWV a concerné l'évaluation de haut-parleurs par analyse temps-fréquence de leur réponse impulsionnelle [E2]. Cette étude a montré, en particulier, l'importance d'une signature temps-fréquence pour décrire, au moins de façon qualitative, des déviations par rapport à ce que devrait être une réponse idéale. Des études complémentaires [E3, E6] ont permis de montrer que le même outil permettait également des mesures quantitatives de distorsion, ce qui peut évidemment se généraliser à toute réponse de filttre ou de système linéaire. On trouvera dans [E4, E5] un exemple d'application de l'approche par DWV pour caractériser la réponse impulsionnelle d'un transducteur ultrasonore et dans [E1] l'analyse d'un filtre à ondes de surface acoustiques par une méthode similaire.

2. Contrôle non-destructif. Le contrôle non-destructif par ultrasons est enfin un domaine d'application potentiel important pour des analyses temps-fréquence $[\mathrm{C} 3, \mathrm{C} 5]$. Les premières applications connues concernent des matériaux composites $[\mathrm{C2}]$ et des polymères $[\mathrm{Cl}, \mathrm{C} 6]$. Dans cette dernière étude, il est à noter que la DWV est utilisée dans deux circonstances différentes: elle permet d'une part d'évaluer une correction de transducteur 
effectuée à l'émission par déconvolution, et d'autre part à estimer une dispersion de vitesse (dans le polymère) qui s'avère caractéristique d'un degré de réticulation. Dans une troisième étude [C4], une méthode non-linéaire (décomposition en valeurs singulières et reconstruction après troncature des valeurs les plus faibles, significatives du bruit), est utilisée pour améliorer l'estimation de la fréquence instantanée d'un écho ultrasonore bruité sans filtrage.

\section{CONCLUSION}

On a vu, d'un point de vue théorique et par l'intermédiaire d'exemples concrets, les avantages que peut présenter une approche temps-fréquence lors de l'analyse de signaux acoustiques non-stationnaires. Un point important à noter est qu'en fait les méthodes temps-fréquence peuvent aller au-delà de l'analyse, comme on a pu le mentionner à diverses reprises. En effet, les propriétés les plus utiles des représentations temps-fréquence (comme l'extraction de caractéristiques de modulation) prennent tout leur sens lorsqu'on peut les utiliser sur des représentations partielles, inaccessibles classiquement par une opération purement temporelle ou purement fréquentielle. Dans le cas d'un problème de séparation de composantes, ceci réalise en fait l'équivalent d'un filtrage dépendant du temps pour lequel le caractère bidimensionnel de la représentation peut être pleinement utilisé. Il en est de même pour les tâches de type décision pour lesquelles on a vu que des formulations tempsfréquence pouvaient être à la fois optimales et riches au niveau de l'interprétation physique. La bonne compréhension actuelle des différents outils disponibles dans quelques cadres théoriques communs (dont une ébauche a été présentée ici) permet d'envisager leur utilisation de manière objective suivant les applications. Elle permet aussi de considérer l'utilité d'outils nouveaux qui peuvent sembler prometteurs, et d'en appréhender plus facilement les mérites potentiels. L'acoustique est à cet égard un champ d'investigation particulièrement important.

\section{REFERENCES}

\section{Théorie}

[T1] ALTES, R.A., J. Acoust. Soc. Am. 67 (1980) 1232-1246.

[T2] CLAASEN, T.A.C.M. et W.F.G. MECKLENBRÄUKER, Philips J. Res. 35 (1980) 217-250, 276300, 372-389.

[T3] COHEN, L., Proc. IEEE 77 (1989) 941-981.

[T4] COMBES, J.M., A. GROSSMANN et PH. TCHAMITCHIAN (Eds.), Wavelets, Springer (1989).

[T5] FLANDRIN, P. et B. ESCUDIE, Trait. Signal 2 (1985) 143-151.

[T6] FLANDRIN, P., Thèse Doct. Etat ès Sc. Phys., Grenoble (1987).

[T7] FLANDRIN, P., IEEE 4th ASSP Workshop on Spectrum Estimation and Modeling (1988) 77-80.

[T8] FLANDRIN, P., IEEE Trans. on Acoust., Speech and Sig. Proc. ASSP-36 (1988) 1377-1384.

[T9] FLANDRIN, P., in [T12] 69-124.

[T10] GRENIER, Y., in [T12] 125-175.

[T11] KCENIG, W., H.K. DUNN et L.Y. LACY, J. Acoust. Soc. Am. 18 (1946) 19-49.

[T12] LONGO, G. et B. PICINBONO $(E d s$.$) , Time and Frequency Representations of Signals and Systems,$ Springer (1989)

[T13] MECKLENBRÄUKER, W.F.G., in [T12] 11-68.

Ondes dispersives, sismique et vibrations

[O1] BOLES, P. et B. BOASHASH, IEEE Int. Conf. on Acoust., Speech and Sig. Proc. ICASSP-87 (1987) 904-907.

[O2] BOASHASH, B. et H.J. WHITEHOUSE, IEEE Int. Symp. on Circ. and Syst. ISCAS-86 (1986) 34-

BOUDREAUX-BARTELS, G.F. et P.J. WISEMAN, IEEE Int. Conf. on Acoust, Speech and Sig. Proc. ICASSP-87 (1987) 2237-2240.

[O4] CHIOLLAZ, M., N. GACHE et P. FLANDRIN, 11ème Coll. GRETSI (1987) 579-582.

[O5] CHIOLLAZ, M. et al., ler CFA (1990).

[O6] FLANDRIN, P., D. GARREAU et C. PUYAL, IEEE Int. Conf. on Acoust., Speech and Sig. Proc. ICASSP-89 (1989) 2246-2249.

[O7] GOUPILLAUD, P., A. GROSSMANN et J. MORLET, Geoexpl. 23 (1984) 85-102.

[O8] SESSAREGO, J.P. et al., en préparation.

Diffusion acoustique

[D1] FLANDRIN, P., J. SAGELOLI, J.P. SESSAREGO et M. ZAKHARIA, Acoust. Lett. 10 (1986) 2328 . 
[D2] FLANDRIN, P., J. SAGELOLI, J.P. SESSAREGO et M. ZAKHARIA, en préparation.

[D3] ZAKHARIA, M., P. FLANDRIN, J. SAGELOLI et J.P. SESSAREGO, J. Acoust. 1 (1988), $185-$ 188.

Milieux réverbérants

[R1] KENNEDY, R.S., Fading Dispersive Communication Channels, Wiley (1969).

[R2] POLLACK, J.D., H. ALRUTZ et M.R. SCHRCEDE, Comptes Rendus II-297 (1983) 21-24,

[R3] POLETTI, M.A., J. Audio. Eng. Soc. 36 (1988) 457-468.

[R4] VERSCHUUR, D.J., A.J.M. KAIZER, W.F. DRUYVESTEYN et D. DE VRIES, J. Audio. Eng. Soc. 36 (1988) 203-212.

[R5] ZHU, W., Acta Acustica 13 (1988) 161-166.

Parole

[P1] D'ALESSANDRO, C. et J.S. LIENARD, IEEE Int. Conf. on Acoust., Speech and Sig. Proc. ICASSP-88 (1988) 351-354.

[P2] CHEN, S.C. et X. YANG, IEEE Int. Conf. on Acoust., Speech and Sig. Proc. ICASSP-88 (1988) 191-194.

[P3] CHESTER, D., F.J. TAYLOR et M. DOYLE, J. Franklin. Inst. 318 (1984) 415-430.

[P4] COHEN, L. et C.A. PICKOVER, IEEE Int. Symp. on Circ. and Syst. ISCAS-86 (1986) $42-45$.

[P5] FRIEDMANN, D.H., IEEE Int. Conf. on Acoust., Speech and Sig. Proc. ICASSP-85 (1985) 11211124.

[P6] GARUDADRI, H., M.P. BEDDOES, J.H.V. GILBERT et A.P. BENGUEREL, IASTED Int. Symp. Appl. Sig. Proc. Dig. Filt. (1985) 196-200.

[P7] LIENARD, J.S., IEEE Int. Conf. on Acoust., Speech and Sig. Proc. ICASSP-87 (1987).

[P8] LOWE, D., M.J. TOMLINSON et R.K. MOORE, IOA Conf. on Speech and Hearing (1986).

[P9] POTTER, R.K., G.A. KOPP et H.C. GREEN, Visible Speech, Van Nostrand (1947).

[P10] RILEY, M.D., Speech Time-Frequency Representations, Kluwer (1989).

[P11] VALIERE, J.C., J.F. ALLARD, C. DEPOLLIER, 11ème Coll. GRETSI (1987) 451-454.

[P12] VELEZ, E.F. et R.G. ABSHER, IEEE Int. Conf. on Acoust., Speech and Signal Proc. ICASSP-89 (1989) 2242-2245.

[P13] VELEZ, E.F. et R.G. ABSHER, IEEE Int. Symp. on Circ. and Syst. ISCAS-89 (1989)

[P14] WOKUREK, W., F. HLAWATSCH et G. KUBIN, Int. Conf. on Dig. Sig. Proc. DSP-84 (1987)

[P15] WOKUREK, W., G. KUBIN et F. HLAWATSCH, 11 th Int. Congr. on Phonetic Sc. ICPhS (1987) 44-47.

[P16] ZUE, V.W., IEEE Int. Conf. on Acoust., Speech and Sig. Proc. ICASSP-86 (1986) 1197-2000.

Acoustique musicale

[M1] ALLARD, J.F., J.C. VALIERE et R. BOURDIER, J. Acoust. Soc. Am. 83 (1988) 1041-1044.

[M2] DORIZE, C., O. RIOUL et A. CHAIGNE, 13ème ICA (1989) 77-80.

[M3] GIBIAT, V., F. WU, P. PERIO et S. CHAINTREUIL, Comptes Rendus II-294 (1982) 633-636.

[M4] KRONLAND-MARTINET, R., J. MORLET et A. GROSSMANN, Int. J. Patt. Recogn. Artif. Intell. 1 (1987) 273-302.

[M5] KRONLAND-MARTINET, R., Computer Music J. (1988) 11-20.

[M6] RODET, X. et P. DEPALLE, 16ème JEP-SFA (1987)

\section{Bioacoustique}

[B1] ALTES, R.A., in : Localization and Orientation in Biology and Engineering (VARJU/SCHNITZLER, eds.), Springer (1984), 234-244.

[B2] ALTES, R.A., SPIE 768 (1987) 61-68.

[B3] ALTES, R.A., in [B10] 725-752.

[B4] FLANDRIN, P., in [B10] 797-802.

[B5] GRENIER, Y., Acustica 61 (1986) 155-165.

[B6] JOHANNESMA, P., A.D. AERTSEN, B. CRANEN et L. VAN ERNING, Hearing Res. 5 (1981) 123-145.

[B7] KISEKA, P.T., communication privée.

[B8] LEROY, Y., C. R. Soc. Biogéogr. (1976) 45-69.

[B9] MARTIN, W. et K. KRÜGER-ALEF, Acustica 61 (1986) 176-183.

[B10] NACHTIGALL, P.E. et P.W.B. MOORE (Eds.), Animal Sonar - Processes and Performance, Plenum (1988)

[B11] SIMMONS, J.A., Cögnition 33 (1989) 155-199. 


\section{Electroacoustique et instrumentation}

[E1] GREENING, R., Ch. KAPPACHER et G. VISINTINI, Ultrasonics Int. (1989),846-851.

[E2] JANSE, C.P. et A.J.M. KAIZER, J. Audio. Eng. Soc. 31 (1983) 198-223.

[E3] JANSE, C.P. et A.J.M. KAIZER, J. Audio. Eng. Soc. 32 (1984) 868-882.

[E4] MARINOVIC, N.N. et W.A. SMITH, IEEE Ultrasonics Symp. (1984) 1023-1028.

[E5] MARINOVIC, N.N. et W.A. SMITH, IEEE Int. Symp. on Circ. and Syst. ISCAS-86 (1986) 50-54.

[E6] PREIS, D., F. HLAWATSCH, P.J. BLOOM et J.A. DEER, J. Audio. Eng. Soc. 35 (1987) 10041012.

Controle non-destructif

[C1] COMBE, P. et M. ZAKHARIA, EUSIPCO-88 (1988) 1337-1340.

[C2] DUMOULIN, J.P. et J.F. de BELLEVAL, Ultrasonics Int. (1985) 769-774.

[C3] FLANDRIN, P., in :Signal Processing and Pattern Recognition in Non-Destructive Evaluation of Materials (C.H. CHEN, ed.), Springer (1988) 109-116.

[C4] MARINOVIC, N.N. et W.A. SMITH, IEEE Ultrasonics Symp. (1985) 841-846.

[C5] ZAKHARIA, M., Ière Ecole d'Hiver sur l'Evaluation Non-Destructive des Matériaux par Ultrasons (1989)

[C6] ZAKHARIA, M., Ultrasonics Int. (1989), 814-819.
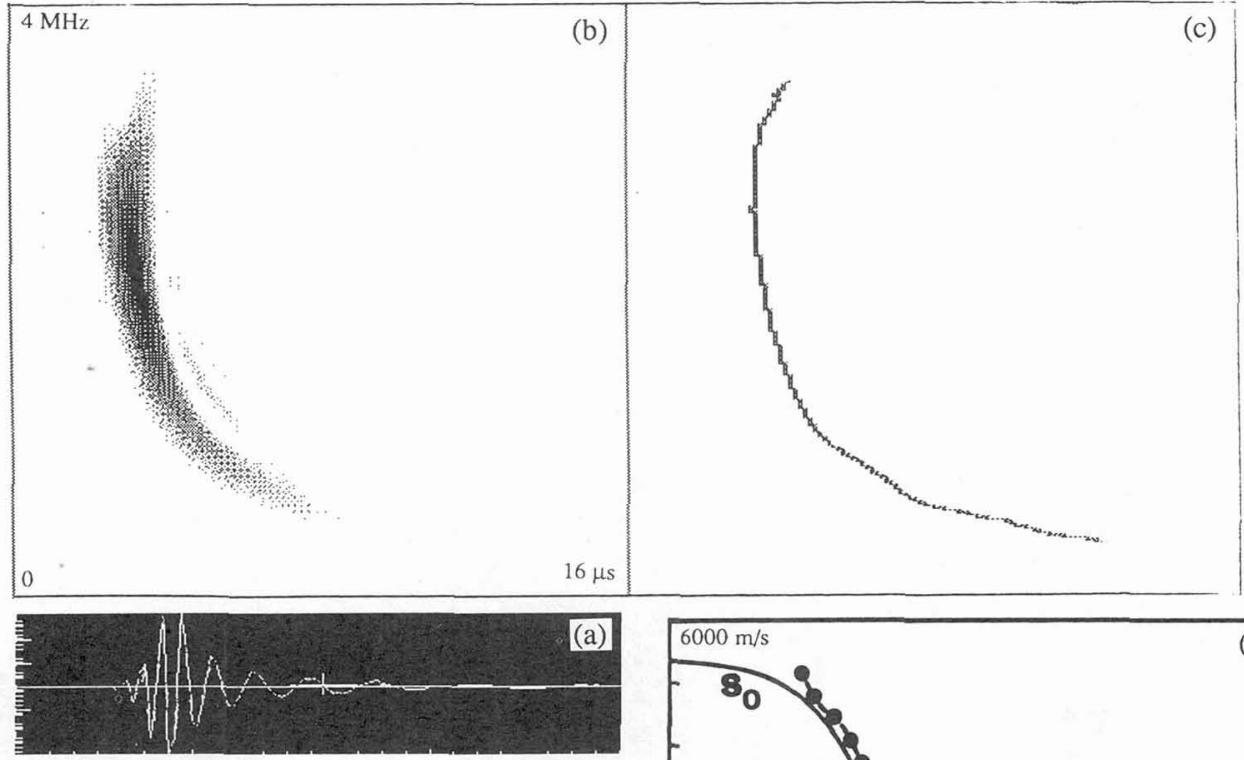

Figure 1 [08]

Onde de Lamb dans une plaque de duraluminium

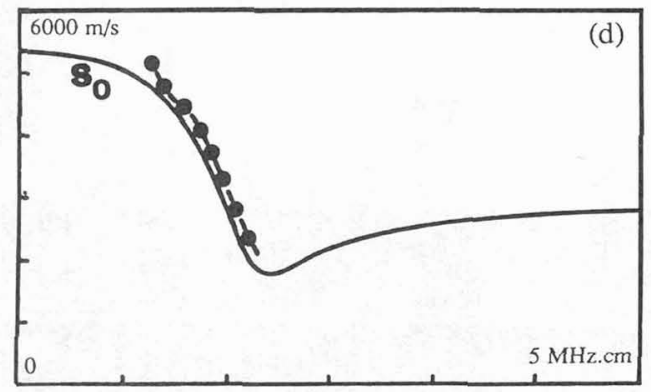

(a) signal expérimental (impulsion après propagation)

(b) DPWVL de (a)

(c) retard de groupe de (a) estimé d'après (b)

(d) dispersion de vitesse du mode de Lamb $S_{0}$ :

- : courbe théorique;

- $\ldots$ : points expérimentaux déduits de (c) 


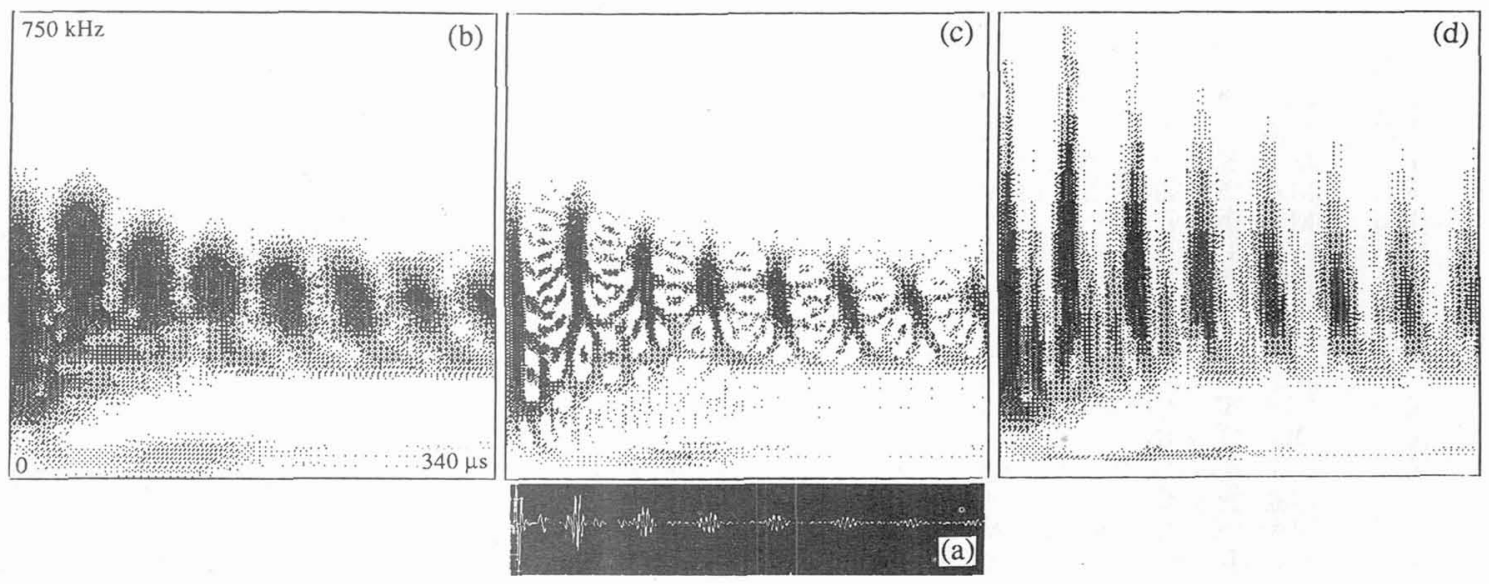

Figure 2 - Rétrodiffusion acoustique d'une coque sphérique mince
(a) réponse impulsionnelle de la coque
(b) spectrogramme de (a)
(c) DPWVL de (a)
(d) scalogramme de (a)
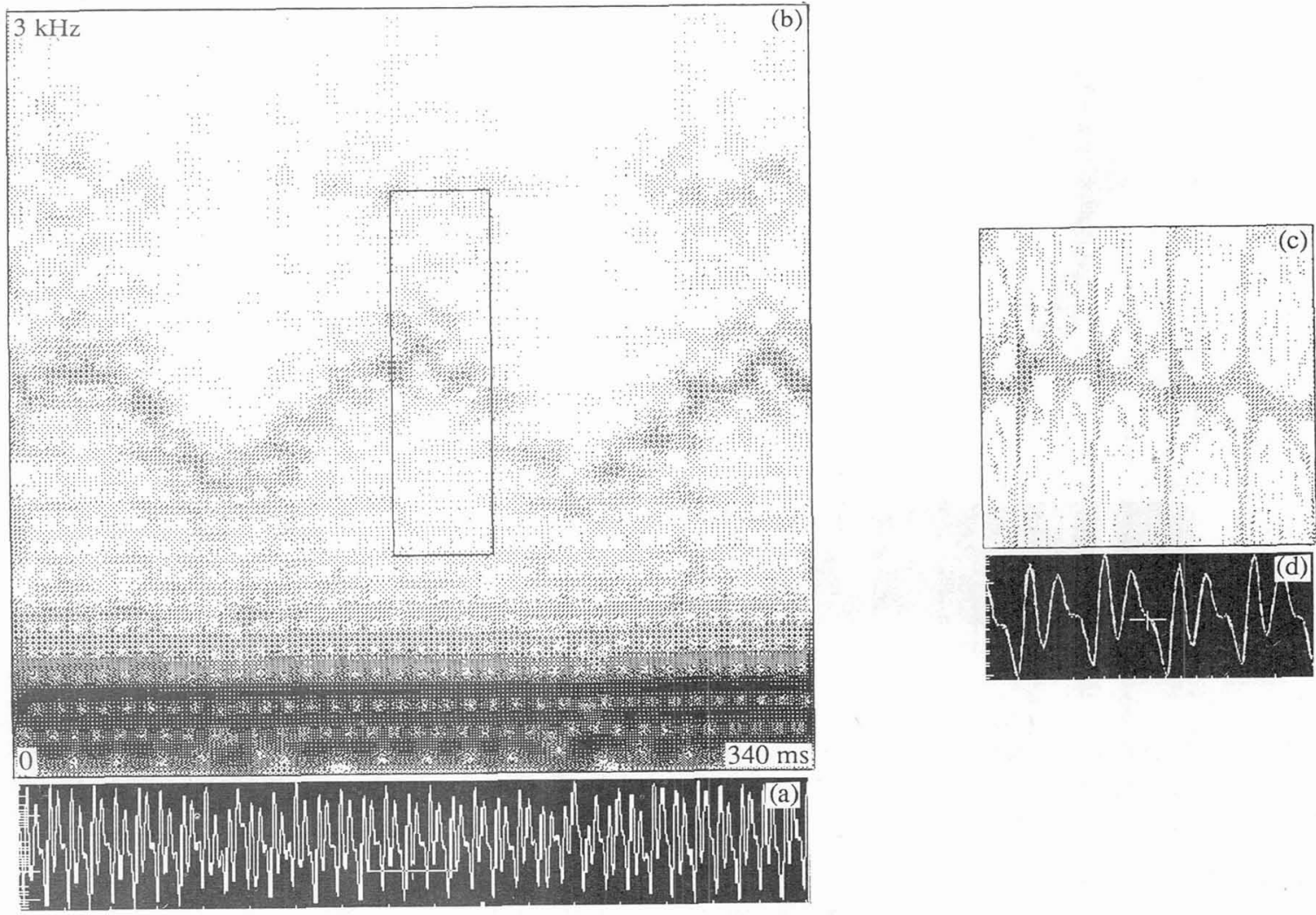

Figure 3 - Un signal de parole

(a) signal de $340 \mathrm{~ms}$ extrait d'une séquence /...uiuiui.../

(b) spectrogramme de (a)

(c) DPWVL calculée sur la zone encadrée de (b)

(d) signal de $21 \mathrm{~ms}$ correspondant (détail de (a)) 